\title{
New inclusion relation of neutrosophic sets with applications and related lattice structure
}

\author{
Xiaohong Zhang ${ }^{1,2} \cdot$ Chunxin $\mathrm{Bo}^{3} \cdot$ Florentin Smarandache $^{4} \cdot$ Jianhua Dai $^{5}$
}

Received: 3 December 2017 / Accepted: 10 April 2018

๑) Springer-Verlag GmbH Germany, part of Springer Nature 2018

\begin{abstract}
The main purpose of this paper is to study the inclusion relations of neutrosophic sets and some applications in multiple attribute decision making. At first, the existing two definitions of inclusion relation (called type-1 and type-2 inclusion relation, respectively) of neutrosophic sets are analyzed, and the deficiencies of type- 1 and type- 2 inclusion relations are illustrated by examples (in fact, they are actually two extreme cases). Second, a new definition of inclusion relation of neutrosophic sets (call it type-3 inclusion relation) is introduced, and a new method of ranking of neutrosophic sets is given. The effectiveness of the ranking method is presented by some application examples in multiple attribute decision making. Finally, type-3 inclusion relation of neutrosophic sets and related lattice structure are investigated in a systematic way, the definitions of type-3 union and type-3 intersection operations are proposed, and the following important result is proved: all of neutrosophic sets based on a certainty domain constitute a generalized De Morgan algebra (non-distributive lattice) with respect to type-3 union, type-3 intersection and complement operations. From this, the essential difference between neutrosophic set and fuzzy set (and intuitionistic fuzzy set) is clarified theoretically.
\end{abstract}

Keywords Neutrosophic set $\cdot$ Fuzzy set $\cdot$ Inclusion relation $\cdot$ Multiple attribute decision $\cdot$ Lattice

\section{Introduction}

Xiaohong Zhang

zhangxiaohong@sust.edu.cn; zhangxh@shmtu.edu.cn

Chunxin Bo

201640311001@stu.shmtu.edu.cn

Florentin Smarandache

smarand@unm.edu

Jianhua Dai

david.joshua@qq.com

1 School of Arts and Sciences, Shaanxi University of Science and Technology, Xi' an 710021, People's Republic of China

2 College of Arts and Sciences, Shanghai Maritime University, Shanghai 201306, People's Republic of China

3 College of Information Engineering, Shanghai Maritime University, Shanghai 201306, People's Republic of China

4 Department of Mathematics, University of New Mexico, Gallup, NM 87301, USA

5 Key Laboratory of High Performance Computing and Stochastic Information Processing (Ministry of Education of China), Hunan Normal University, Changsha 410081, Hunan, People's Republic of China
The notion of neutrosophic set is proposed by Smarandache in 1998 (see [20-22]). Now, neutrosophic set is gaining significant attention in solving many real life problems that involve uncertainty, impreciseness, incompleteness, inconsistent, and indeterminacy. The theory of netrosophic set has been applied in multiple attribute decision making, computational intelligence, machine learning, image processing, medical diagnosis, fault diagnosis, optimization design, algebraic systems and so on (see $[2,7,8,11-17,28-33$, 35-39]). Moreover, a lot of new neutrosophic theories have been proposed, for examples, neutrosophic cubic set (see $[9,10,34])$, neutrosophic soft set and neutrosophic rough set (see $[3,6,25])$.

The core idea of neutrosophic theory is to describe the uncertainty of concepts from three mutually independent aspects. In fact, this idea has many different forms of expression in the academic circle. For examples: (1) from the rough set, interval set, granular computing and other research nutrition, the famous scholar Yao creatively put forward three-way decision theory, has become a hot research topic in related fields (see [26, 27]). A very interesting research 
trend is that the connection between neutrosophic theory and three-way decision theory is concerned in [18]. (2) A picture fuzzy set (proposed by Cuong, see [1, 4-6]) can be regarded as a special neutrosophic set, so relevant research can be incorporated into the framework of neutrosophic theory. In fact, the notion of picture fuzzy set is called standard neutrosophic set in [6]. (3) Wang et al. introduced the new notions of ternary fuzzy set and ternary fuzzy number in [23]; by simple comparison, we find that the two concepts of picture fuzzy set and ternary fuzzy set are essentially equivalent. We believe that the integration of these theories will provide more effective methods and tools for solving uncertainty problems.

In the neutrosophic set, truth-membership, indeterminacy-membership, and falsity-membership are represented independently. If $U$ is a set, a neutrosophic set defined on the universe $U$ assigns to each element $x \in U$, a triple $(T(x), I(x), F(x))$, where $T(x), I(x)$ and $F(x)$ are standard or non-standard elements of non-standard unit interval ] $0^{-}, 1^{+}\left[=0^{-} \bigcup[0.1] \bigcup 1^{+} . T\right.$ is the degree of truth-membership in the set $U, I$ is the degree of indeterminacy-membership in the set $U$ and $F$ is the degree of non-membership in the set $U$. In this paper we work with special neutrosophic sets that their degrees of memberships are standard real numbers in $[0,1]$, they are called single valued neutrosophic set (see [24]). It can be seen from this, neutrosophic set is a powerful general formal framework that generalizes the concepts of fuzzy set and intuitionistic fuzzy set.

For a set theory, inclusion relation is a basic concept; it plays a fundamental role in defining the union and intersection operations between sets, as well as the basis for related applications. From the existing literature, there are two different definitions of the inclusion relation of neutrosophic sets. In this paper, we gave some examples to illustrate the shortcomings of the two inclusion relations, and then propose a new inclusion relation (call it type-3 inclusion relation). Based on type- 3 inclusion relation, we establish a new ranking method of neutrosophic sets and apply it to multiple attribute decision making problems. At the same time, this paper also studies the union and intersection operations corresponding to type-3 inclusion relation and the related lattice structure. Using these research results, we reveal the essential difference between neutrosophic set and fuzzy set (interval-valued fuzzy set, intuitionistic fuzzy set, and so on) from an algebraic point of view.

\section{Neutrosophic sets and type-1 (type-2) inclusion relations}

Definition 2.1 [20, 21] Let $X$ be a space of points (objects), with a generic element in $X$ denoted by $x$. A neutrosophic set $A$ in $X$ is characterized by a truth-membership function
$T_{A}(x)$, an indeterminacy-membership function $I_{A}(x)$, and a falsity- membership function $F_{A}(x)$. The functions $T_{A}(x)$, $I_{A}(x)$, and $F_{A}(x)$ are real standard or non-standard subsets of ]$^{-} 0,1^{+}\left[\text {. That is, } T_{A}(x): X \rightarrow\right]^{-} 0,1^{+}\left[, I_{A}(x): X \rightarrow\right]^{-} 0,1^{+}[$, and $\left.F_{A}(x): X \rightarrow\right]^{-} 0,1^{+}[$. Thus, there is no restriction on the sum of $T_{A}(x), I_{A}(x)$, and $F_{A}(x)$, so ${ }^{-} 0 \leq \sup T_{A}(x)+\sup I_{A}(x)$ $+\sup F_{A}(x) \leq 3^{+}$.

Definition 2.2 [24] Let $X$ be a space of points (objects) with generic elements in $X$ denoted by $x$. A single valued neutrosophic set $A$ in $X$ is characterized by truth-membership function $T_{A}(x)$, indeterminacy-membership function $I_{A}(\mathrm{x})$, and falsity-membership function $F_{A}(x)$. Then, a single valued neutrosophic set $A$ can be denoted by

$A=\left\{\left\langle x, T_{A}(x), I_{A}(x), F_{A}(x)\right\rangle \mid x \in X\right\}$,

where $T_{A}(x), I_{A}(x), F_{A}(x) \in[0,1]$ for each point $x$ in $X$. Therefore, the sum of $T_{A}(x), I_{A}(x)$, and $F_{A}(x)$ satisfies the condition $0 \leq T_{A}(x)+I_{A}(x)+F_{A}(x) \leq 3$.

For convenience, "single valued neutrosophic set" is abbreviated to "neutrosophic set" later. In this paper, we use the symbol $N S(X)$ to denote the set of all single valued neutrosophic set in $X$.

Two single valued neutrosophic sets $A$ and $B$ are equal, written as $A=B$, if and only if $T_{A}(x)=T_{B}(x), I_{A}(x)=I_{B}(x)$ and $F_{A}(x)=F_{B}(x)$ for any $x$ in $X$.

For the inclusion relation of neutrosophic sets, there are two different definitions in the literature. An original definition is proposed by Smarandache (see [20-22]), we call it type-1 inclusion relation in this paper, denote it by $\subseteq_{1}$; another one is used in some papers (see [2, 3, 24]), we call it type- 2 inclusion relation in this paper, denote it by $\subseteq_{2}$.

Definition 2.3 [20-22] A single valued neutrosophic set $A$ is contained in the other single valued neutrosophic set $B$, denote $A \subseteq_{1} B$, if and only if $T_{A}(x) \leq T_{B}(x), I_{A}(x) \geq I_{B}(x)$, $F_{A}(x) \geq F_{B}(x)$ for any $x$ in $X$.

It is easy to verify that the above inclusion relation $\subseteq_{1}$ is a partial ordered relation on $N S(X)$. We called it type-1 inclusion relation in this paper.

Definition 2.4 [2, 3, 24] A single valued neutrosophic set $A$ is contained in the other single valued neutrosophic set $B$, denote $A \subseteq_{2} B$, if and only if $T_{A}(x) \leq T_{B}(x), I_{A}(x) \leq I_{B}(x)$, $F_{A}(x) \geq F_{B}(x)$ for any $x$ in $X$.

Obviously, the above inclusion relation $\subseteq_{2}$ is also a partial ordered relation on $N S(X)$. We called it type- 2 inclusion relation in this paper. 
Proposition 2.1 Two single valued neutrosophic sets $A$ and $B$ are equal, if and only if $A \subseteq_{1} B$ and $B \subseteq_{1} A$, if and only if $A \subseteq_{2} B$ and $B \subseteq_{2} A$.

For set theory, union and intersection operations are corresponding to inclusion relation. Therefore, there are two kinds of union and intersection operations corresponding to the two inclusion relations mentioned above. Interestingly, the complement operation uses the same definition in this paper (in fact, there are indeed different ways of defining complement operation in the literature, and we will discuss this problem in another paper). The basic definitions of these concepts are listed as follows.

Definition 2.5 [20-22] The type-1 union of two neutrosophic sets $A$ and $B$ is a neutrosophic set $C$, written as $C=A \cup_{1} B$, whose truth-membership, indeterminacy-membership and falsity-membership functions are related to those of $A$ and $B$ by

$T_{C}(x)=\max \left(T_{A}(x), T_{B}(x)\right), I_{C}(x)=\min \left(I_{A}(x), I_{B}(x)\right)$,

$F_{C}(x)=\min \left(F_{A}(x), F_{B}(x)\right), \forall x \in X$.

Definition 2.6 $[2,3,24]$ The type-2 union of two neutrosophic sets $A$ and $B$ is a neutrosophic set $C$, written as $C=A \cup_{2} B$, whose truth-membership, indeterminacy-membership and falsity-membership functions are related to those of $A$ and $B$ by

$T_{C}(x)=\max \left(T_{A}(x), T_{B}(x)\right), I_{C}(x)=\max \left(I_{A}(x), I_{B}(x)\right)$,

$F_{C}(x)=\min \left(F_{A}(x), F_{B}(x)\right), \forall \in X$.

Definition 2.7 [20-22] The type-1 intersection of two neutrosophic sets $A$ and $B$ is a neutrosophic set $D$, written as $D=A \cap_{1} B$, whose truth-membership, indeterminacymembership and falsity-membership functions are related to those of $A$ and $B$ by

$T_{D}(x)=\min \left(T_{A}(x), T_{B}(x)\right), I_{D}(x)=\max \left(I_{A}(x), I_{B}(x)\right)$,

$F_{D}(x)=\max \left(F_{A}(x), F_{B}(x)\right), \forall \in X$.

Definition 2.8 $[2,3,24]$ The type-2 intersection of two neutrosophic sets $A$ and $B$ is a neutrosophic set $D$, written as $D=A \cap_{2} B$, whose truth-membership, indeterminacymembership and falsity-membership functions are related to those of $A$ and $B$ by

$T_{D}(x)=\min \left(T_{A}(x), T_{B}(x)\right), I_{D}(x)=\min \left(I_{A}(x), I_{B}(x)\right)$,

$F_{D}(x)=\max \left(F_{A}(x), F_{B}(x)\right), \forall \in X$.

Definition 2.9 The complement of a neutrosophic set $A$ is denote by $A^{\mathrm{c}}$, is defined as
$T_{A^{c}}(x)=F_{A}(x), I_{A^{c}}(x)=1-I_{A}(x), F_{A^{c}}(x)=T_{A}(x), \forall \in X$.

We can prove the following important proposition (the proof is omitted).

Definition 2.10 [19] A generalized De Morgan algebra (or GM-algebra) is a universal algebra $(M, \vee, \wedge, 0,1)$ where $(M, \wedge, \vee, 0,1)$ is a bounded lattice and the unary operation satisfies the identities:

GM1 : $x=\left(x^{-}\right)^{-}$

$\mathrm{GM} 2:(x \wedge y)^{-}=x^{-} \vee y^{-}$;

GM3 $: 1^{-}=0$.

For a generalized De Morgan algebra $\left(M, \wedge, \vee,{ }^{-}, 0,1\right)$, if $(M, \wedge, \vee, 0,1)$ is a bounded distributive lattice, then $(M, \vee, \wedge,-, 0,1)$ is a De Morgan algebra.

Proposition 2.2 Let $X$ be a universe. Then $\left(N S(X), \subseteq_{1}, \cup_{1}\right.$, $\left.\cap_{1},{ }^{c},(0,1,1),(1,0,0)\right)$ is a De Morgan algebra, and $(N S(X)$, $\left.\subseteq_{2}, \cup_{2}, \cap_{2},{ }^{c},(0,0,1),(1,1,0)\right)$ is also a De Morgan algebra.

\section{Type-3 inclusion relation of neutrosophic sets}

First, we analyze the inadequacies of the type- 1 and type- 2 inclusion relations of neutrosophic sets.

For any $A, B \in N S(X)$,

$A=\left\{\left\langle x, T_{A}(x), I_{A}(x), F_{A}(x)\right\rangle \mid x \in X\right\} ;$

$B=\left\{\left\langle x, T_{B}(x), I_{B}(x), F_{B}(x)\right\rangle \mid x \in X\right\}$.

By Definition 2.3 and 2.4, we know that

$A \subseteq_{1} B \Rightarrow T_{A}(x) \leq T_{B}(x), I_{A}(x) \geq I_{B}(x), F_{A}(x) \geq F_{B}(x), \forall x \in X$. $A \subseteq_{2} B \Rightarrow T_{A}(x) \leq T_{B}(x), I_{A}(x) \leq I_{B}(x), F_{A}(x) \geq F_{B}(x), \forall x \in X$.

Thus,

$A \subseteq_{1} B \Rightarrow T_{A}(x) \leq T_{B}(x), I_{A}(x)+F_{A}(x) \geq I_{B}(x)+F_{B}(x), \forall x \in X$. $A \subseteq_{2} B \Rightarrow T_{A}(x)+I_{A}(x) \leq T_{B}(x)+I_{B}(x), F_{A}(x) \geq F_{B}(x), \forall x \in X$.

This means that type- 1 and type- 2 inclusion relations are actually divided three membership functions into two groups, and then determines the order relation using the method similar to intuitionistic fuzzy sets; the difference between the two types is that one calculates the uncertain part in the truth-part and the other is to calculate the uncertain part in the falsity-part. In other words, the two inclusion relations do not really take advantage of the three membership functions; they are actually two extreme ways of handling. From another point of view, since $I(x)$ 
represent indeterminacy, it should not be added to the $T(x)$ or $F(x)$.

Now, we give following example to illustrate the inadequacies of the two inclusion relations.

Example 3.1 Let $X$ be a universe, $A, B, C, D \in N S(X)$. If

$T_{A}(x)=0.5, I_{A}(x)=0.4, F_{A}(x)=0.1, \forall x \in X ;$

$T_{B}(x)=0.5, I_{B}(x)=0.3, F_{B}(x)=0.1, \forall x \in X$;

$T_{C}(x)=0.6, I_{C}(x)=0.1, F_{C}(x)=0.25, \forall x \in X$;

$T_{D}(x)=0.6, I_{D}(x)=0.09, F_{D}(x)=0.1, \forall x \in X$.

Then

$A \subseteq_{1} B$, but $B \subseteq_{2} A$;

$C \subseteq_{1} D$, but $C \subsetneq_{2} D$ and $D \subsetneq_{2} C$.

Assuming that the three membership functions in the neutrosophic sets are understood as the proportion of the votes in the voting process that are agreed, neutral (indeterminacy) or against votes, the above results show that type-1 and type- 2 inclusion relations are not reasonable:

1. $T_{A}(x)=T_{B}(x)=0.5, F_{A}(x)=F_{A}(x)=0.1$, and the number of neutral, $I_{A}(x)=0.4>0.3=I_{B}(x)$, why $A \subseteq_{1} B$ ? This is unjustifiable, it follows that the definition of type-1 inclusion relation is inadequate;

2. $T_{C}(x)=T_{D}(x)=0.6, F_{C}(x)=0.25>0.1=F_{D}(x)$, and the difference between $I_{C}(x)$ and $I_{D}(x)$ is very small. Intuitively, $C$ is less than $D$, but $C$ and $D$ can not be compared by using $\subseteq_{2}$, it follows that the definition of type- 2 inclusion relation is inadequate.

In order to overcome the shortcomings of the above two kinds of inclusion relations, we introduce type- 3 inclusion relation. The main idea is to give full play to the three membership functions and subdivide various possible situations, it can be regarded as the generalization of related methods in $[1,5]$.

Definition 3.1 Let $A$ and $B$ be two neutrosophic sets in the universe $X$. The type-3 inclusion relation $\subseteq_{3}$ is defined as follows: $A \subseteq_{3} B$ if and only if

$\forall x \in X,\left(T_{A}(x)<T_{B}(x), F_{A}(x) \geq F_{B}(x)\right)$,

or $\left(T_{A}(x)=T_{B}(x), F_{A}(x) F_{B}(x)\right)$,

or $\left(T_{A}(x)=T_{B}(x), F_{A}(x)=F_{B}(x)\right.$ and $\left.I_{A}(x) \leq I_{B}(x)\right)$.
For Example 3.1, we can get that $B \subseteq_{3} A$ and $C \subseteq_{3} D$.

The following unconventional voting example shows that the three independent membership functions in neutrosophic sets have practical application background.

Example 3.2 Take a voting of a region's deputies for example, where the voting rules are special, which allows voters to choose multiple options at the same time. Assume that there are three candidates $(A, B, C)$ determined, and two deputies should be chosen. The provisions of the voting are given as follows:

1. For each candidate can choose in favor (draw $\sqrt{ }$ ), neutral (draw $\bigcirc$ ), against (draw $\times$ );

2. You can select multiple options;

3. Do not mark with any symbol if abstain from voting, that is, you may not choose any option for any candidate.

In the end, there are 100 valid ballots. The final vote count of candidate is given as follows:

A: 75 people vote for, 40 people neutral, 20 people against

B: 40 people vote for, 25 people neutral, 50 people against

C: 60 people vote for, 60 people neutral, 75 people against

We used three neutrosophic sets to represent the voting results, that is, $A, B$ and $C$ are regarded as neutrosophic sets in the single point set $X=\left\{x_{0}\right.$ :ideal candidate $\}$ :

$T_{A}\left(x_{0}\right)=0.75, I_{A}\left(x_{0}\right)=0.4, F_{A}\left(x_{0}\right)=0.2$;

$T_{B}\left(x_{0}\right)=0.6, I_{B}\left(x_{0}\right)=0.25, F_{B}\left(x_{0}\right)=0.5$;

$T_{C}\left(x_{0}\right)=0.6, I_{C}\left(x_{0}\right)=0.6, F_{C}\left(x_{0}\right)=0.75$

By Definition 3.2, we have

$B \subseteq_{3} A, C \subseteq_{3} A$, and $C \subseteq_{3} B$.

Then the candidates $A$ and $B$ win two of the three qualifications.

Proposition 3.1 Let $X$ be a universe, $A, B, C \in N S(X)$. Then, (1) $A \subseteq_{3} A$; (2) $\left(A \subseteq_{3} B, B \subseteq_{3} A\right) \Rightarrow A=B$; (3) $\left(A \subseteq_{3} B, B \subseteq_{3}\right.$ C) $\Rightarrow A \subseteq_{3} C$.

Definition 3.2 Let $A$ and $B$ be neutrosophic sets in $X$. The type- 3 union, type- 3 intersection and complement are defined as follows: 
1. $A \cup_{3} B= \begin{cases}A=\left\{\left(x, T_{A}(x), I_{A}(x), F_{A}(x)\right) \mid x \in X\right\}, & \text { if } B \subseteq_{3} A \\ B=\left\{\left(x, T_{B}(x), I_{B}(x), F_{B}(x)\right) \mid x \in X\right\}, & \text { if } A \subseteq_{3} B \\ \left\{\left(x, \max \left(T_{A}(x), T_{B}(x)\right), 0, \min \left(F_{A}(x), F_{B}(x)\right)\right) \mid x \in X\right\}, & \text { otherwise }\end{cases}$

2. $A \cap_{3} B= \begin{cases}A=\left\{\left(x, T_{A}(x), I_{A}(x), F_{A}(x)\right) \mid x \in X\right\}, & \text { if } A \subseteq_{3} B \\ B=\left\{\left(x, T_{B}(x), I_{B}(x), F_{B}(x)\right) \mid x \in X\right\}, & \text { if } B \subseteq_{3} A \\ \left\{\left(x, \min \left(T_{A}(x), T_{B}(x)\right), 1, \max \left(F_{A}(x), F_{B}(x)\right)\right) \mid x \in X\right\}, & \text { otherwise }\end{cases}$

3. $\left.A^{c}=\left\{\left(x, F_{A}(x), 1-I_{A}(x), T_{A}(x)\right) \mid x \in X\right)\right\}$.

It is easy to verify the following conclusions (the proofs are omitted here, in Sect. 5, we will give the proof in a more general framework).

Proposition 3.2 Let A, B be neutrosophic sets in $X$, then

1. $A \cup_{3} B$ is the least upper bound (supremum) of $A$ and $B$ under the order relation $\subseteq_{3}$;

2. $A \cap_{3} B$ is the greatest lower bound (infimum) of $A$ and $B$ under the order relation $\subseteq_{3}$.

Proposition 3.3 Let $A, B, C$ be neutrosophic sets in $X$, then

1. $A \cap_{3} A=A, A \cup_{3} A=A$;

2. $A \cap_{3} B=B \cap_{3} A, A \cup_{3} B=B \cup_{3} A$;

3. $\left(A \cap_{3} B\right) \cap_{3} C=A \cap_{3}\left(B \cap_{3} C\right),\left(A \cup_{3} B\right) \cup_{3} C=A \cup_{3}\left(B \cup_{3} C\right)$;

4. $A \cap_{3}\left(B \cup_{3} A\right)=A, A \cup_{3}\left(B \cap_{3} A\right)=A$;

5. $A \subseteq_{3} B \Leftrightarrow A \cup_{3} B=B ; A \subseteq_{3} B \Leftrightarrow A \cap_{3} B=A$.

Proposition 3.4 Let $A$ and $B$ be neutrosophic sets in $X$, then

1. $\left(A^{c}\right)^{c}=A$;

2. $\left(A \cap_{3} B\right)^{c}=A^{c} \cup_{3} B^{c}$;

3. $\left(A \cup_{3} B\right)^{c}=A^{c} \cap_{3} B^{c}$.

Theorem 3.1 Let NS(X) be the set of all neutrosophic sets in universe $X$. Denote

$0_{\text {type-3 }}=\{(x, 0,0,1) \mid x \in X\}$,

$1_{\text {type-3 }}=\{(x, 1,1,0) \mid x \in X\}$.

Then $\left(N S(X), \cup_{3}, \cap_{3},{ }^{c}, 0_{\text {type- } 3}, 1_{\text {type- } 3}\right)$ is a generalized De Morgan algebra.

\section{A new ranking method with applications}

The inclusion relations of neutrosophic sets are closely related to the ranking methods of the neutrosophic numbers, and the ranking methods play a basic important role in the application of neutrosophic sets in multi attribute decision making. In [22], using three functions: neutrosophic score function, neutrosophic accuracy function, and neutrosophic certainty function, Smarandache define a total order on the set of neutrosophic numbers. In this section, a new ranking method will be given with the help of the idea of type-3 inclusion relation in Sect. 3 .

As a preparation, the concept of neutrosophic number and the order relation on it will be introduced.

If $t, i, f \in[0,1]$, then $(t, i, f)$ is called a single-valued neutrosophic number. Similar to [5], we consider the set $D^{*}$ defined by

$D^{*}=\left\{x=\left(x_{1}, x_{2}, x_{3}\right) \mid x_{1}, x_{2}, x_{3} \in[0,1]\right\}$.

From now on, we will assume that if $x, y \in D^{*}$ or $(t, i, f) \in D^{*}$, then $x_{1}$ (or $\left.t\right), x_{2}$ (or $i$ ) and $x_{3}$ (or $f$ ) denote, respectively, the first, the second and the third component. We denote the units of $D^{*}$ by $1_{D^{*}}=(1,1,0)$ and $0_{D^{*}}=(0,0$, $1)$, respectively.

Obviously, for every neutrosophic set

$A=\left\{\left(x, T_{A}(x), I_{A}(x), F_{A}(x)\right) \mid x \in X\right\}$,

It correspond with an $D^{*}$-fuzzy set, i.e., a mapping

$A: X \rightarrow D^{*}: x \mapsto\left(T_{A}(x), I_{A}(x), F_{A}(x)\right)$.

By Definition 2.3 and 2.4, the type-1 and type- 2 inclusion relation of neutrosophic sets are based on the following order relation on $D^{*}$ :

$\forall x, y \in D^{*}, x \leq_{1} y \Leftrightarrow\left(x_{1} \leq y_{1}\right)$ and $\left(x_{2} \geq y_{2}\right)$ and $\left(x_{3} \geq y_{3}\right)$.

$\forall x, y \in D^{*}, x \leq_{2} y \Leftrightarrow\left(x_{1} \leq y_{1}\right)$ and $\left(x_{2} \leq y_{2}\right)$ and $\left(x_{3} \geq y_{3}\right)$.

By Definition 3.1, type-3 inclusion relation is based on the following order relation on $D^{*}$ :

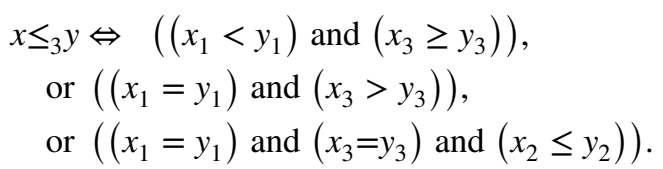

Remark 4.1 In next section, we will give a rigorous proof of the lattice properties of type-3 order relation on $D^{*}$. Note that, if for $x, y \in D^{*}$ that neither $x \leq_{3} y$ nor $y \leq_{3} x$, then $x$ and $y$ are incomparable, denoted as $x \|_{\leq_{3}} y$. Similar symbols are also applicable to type- 1 and type- 2 order relations. 
Definition 4.1 Let $(t, i, f)$ be a single-valued neutrosophicnumber. The type-3 score function is defined by

$s: D^{*} \rightarrow[0,1], s(t, i, f)=\frac{t+(1-f)}{2}$.

The type- 3 accuracy function is defined by

$h: D^{*} \rightarrow[0,1], h(t, i, f)=\frac{t}{t+(1-f)}$.

It is easy to verify the following conclusion which shows that type- 3 score function and type- 3 ordered relation are compatible.

Proposition 4.1 Let $\left(t_{1}, i_{1}, f_{1}\right)$ and $\left(t_{2}, i_{2}, f_{2}\right)$ be two singlevalued neutrosophic numbers. Then

$\left(t_{1}, i_{1}, f_{1}\right) \leq_{3}\left(t_{2}, i_{2}, f_{2}\right) \Rightarrow s\left(t_{1}, i_{1}, f_{1}\right) \leq s\left(t_{2}, i_{2}, f_{2}\right)$.

It is easy to verify the following conclusion which shows that type-3 score function and type-3 accuracy function determine the 1, 3 component of the neutrosophic number (not related to the second component).

Proposition 4.2 Let $\left(t_{1}, i_{1}, f_{1}\right)$ and $\left(t_{2}, i_{2}, f_{2}\right)$ be two singlevalued neutrosophic numbers. Then

$$
\begin{aligned}
s\left(t_{1}, i_{1}, f_{1}\right) & =s\left(t_{2}, i_{2}, f_{2}\right) \text { and } h\left(t_{1}, i_{1}, f_{1}\right)=h\left(t_{2}, i_{2}, f_{2}\right) \\
& \Rightarrow\left(t_{1}=t_{2}, f_{1}=f_{2}\right) .
\end{aligned}
$$

Now, we propose a new ranking method of the neutrosophic numbers.

Definition 4.2 Let $\left(t_{1}, i_{1}, f_{1}\right)$ and $\left(t_{2}, i_{2}, f_{2}\right)$ be two singlevalued neutrosophic numbers. Then

1. if $s\left(t_{1}, i_{1}, f_{1}\right)<s\left(t_{2}, i_{2}, f_{2}\right)$, then $\left(t_{1}, i_{1}, f_{1}\right)<\left(t_{2}, i_{2}, f_{2}\right)$;

2. if $s\left(t_{1}, i_{1}, f_{1}\right)=s\left(t_{2}, i_{2}, f_{2}\right), h\left(t_{1}, i_{1}, f_{1}\right)<h\left(t_{2}, i_{2}, f_{2}\right)$, then $\left(t_{1}, i_{1}, f_{1}\right)<\left(t_{2}, i_{2}, f_{2}\right)$

3. if $s\left(t_{1}, i_{1}, f_{1}\right)=s\left(t_{2}, i_{2}, f_{2}\right), h\left(t_{1}, i_{1}, f_{1}\right)=h\left(t_{2}, i_{2}, f_{2}\right)$, $i_{1}<i_{2}$, then $\left(t_{1}, i_{1}, f_{1}\right) \prec\left(t_{2}, i_{2}, f_{2}\right) ; i_{1}=i_{2}$, then $\left(t_{1}, i_{1}, f_{1}\right)=$ $\left(t_{2}, i_{2}, f_{2}\right)$.

It is easy to verify the following conclusions which show that the relation $\preccurlyeq(<$ or $=)$ is a total order on $D^{*}$.

Proposition 4.3 For any $\left(t_{1}, i_{1}, f_{1}\right),\left(t_{2}, i_{2}, f_{2}\right)$, and $\left(t_{3}, i_{3}, f_{3}\right)$ in $D^{*}$, the following assertions hold:

1. $\left(t_{1}, i_{1}, f_{1}\right) \leqslant\left(t_{1}, i_{1}, f_{1}\right)$;

2. if $\left(t_{1}, i_{1}, f_{1}\right) \leqslant\left(t_{2}, i_{2}, f_{2}\right)$ and $\left(t_{2}, i_{2}, f_{2}\right) \leqslant\left(t_{1}, i_{1}, f_{1}\right)$, then $\left(t_{1}\right.$, $\left.i_{1}, f_{1}\right)=\left(t_{2}, i_{2}, f_{2}\right)$;

3. if $\left(t_{1}, i_{1}, f_{1}\right) \leqslant\left(t_{2}, i_{2}, f_{2}\right)$ and $\left(t_{2}, i_{2}, f_{2}\right) \leqslant\left(t_{3}, i_{3}, f_{3}\right)$, then ( $t_{1}$, $\left.i_{1}, f_{1}\right) \leqslant\left(t_{3}, i_{3}, f_{3}\right)$;

4. if $\left(t_{1}, i_{1}, f_{1}\right) \nless\left(t_{2}, i_{2}, f_{2}\right)$, then $\left(t_{2}, i_{2}, f_{2}\right) \prec\left(t_{1}, i_{1}, f_{1}\right)$.
Example 4.1 Let us consider two single-valued neutrosophic numbers:

$\alpha=(0.6,0.1,0.4), b=(0.5,0.1,0.3)$.

By Definition 4.1 and 4.2, we have

$s(\alpha)=0.6, s(\beta)=0.6$;

$h(\alpha)=0.5, h(\beta)=0.417$.

Hence, $\beta<\alpha$. This is in accordance with the conclusion by using neutrosophic score function, neutrosophic accuracy function, and neutrosophic certainty function in [22] (see Page 23, 24 in second extended and improved edition).

Now, we discuss some examples for multi-criteria decision-making problem of engineering alternatives by using the above ranking method of the neutrosophic numbers.

Example 4.2 Let us consider the decision-making problem adapted from [31]. There is an investment company, which wants to invest a sum of money in the best option. There is a panel with four possible alternatives to invest the money: (1) $A_{1}$ is a car company; (2) $A_{2}$ is a food company; (3) $A_{3}$ is a computer company; (4) $A_{4}$ is an arms company. The investment company must take a decision according to the following three criteria: (1) $C_{1}$ is the risk; (2) $C_{2}$ is the growth; (3) $C_{3}$ is the environmental impact. Then, the weight vector of the criteria is given by $W=(0.35,0.25,0.4)$.

For the evaluation of an alternative $A_{i}(i=1,2,3,4)$ with respect to a criterion $C_{j}(j=1,2,3)$, it is obtained from the questionnaire of a domain expert. For example, when we ask the opinion of an expert about an alternative $A_{1}$ with respect to a criterion $C_{1}$, he or she may say that the possibility in which the statement is good is 0.4 and the statement is poor is 0.3 and the degree in which he or she is not sure is 0.2 . For the neutrosophic notation, it can be expressed as $\alpha_{11}=(0.4,0.2,0.3)$. Thus, when the four possible alternatives with respect to the above three criteria are evaluated by the expert, we can obtain the following simplified neutrosophic decision matrix $D$.

In [31], the author obtains the weighted arithmetic average value (aggregating simplified neutrosophic value) $\alpha_{i}$ for $A_{i}(i=1,2,3,4)$ :

$\alpha_{1}=(0.3268,0.2000,0.3881)$,

$\alpha_{2}=(0.5627,0.1414,0.2000)$,

$\alpha_{3}=(0.4375,0.2416,0.2616)$,

$\alpha_{4}=(0.5746,0.1555,0.1663)$.

$D=\left[\begin{array}{lll}(0.4,0.2,0.3) & (0.4,0.2,0.3) & (0.2,0.2,0.5) \\ (0.6,0.1,0.2) & (0.6,0.1,0.2) & (0.5,0.2,0.2) \\ (0.3,0.2,0.3) & (0.5,0.2,0.3) & (0.5,0.3,0.2) \\ (0.7,0.0,0.1) & (0.6,0.1,0.2) & (0.4,0.3,0.2)\end{array}\right]$ 
By Definition 4.1 and 4.2, we have

$s\left(\alpha_{1}\right)=0.4694, \quad s\left(\alpha_{2}\right)=0.6814, \quad s\left(\alpha_{3}\right)=0.5880$, $s\left(\alpha_{4}\right)=0.7042$;

$\alpha_{1}<\alpha_{3}<\alpha_{2}<\alpha_{4}$.

Therefore, the alternative $A_{4}$ is the best choice among all the alternatives.

Moreover, by the weighted geometric average operator, we can obtain the following computational results:

$\alpha_{1}=(0.3031,0.2000,0.3680)$,

$\alpha_{2}=(0.5578,0.1320,0.2000)$,

$\alpha_{3}=(0.4181,0.2352,0.2551)$,

$\alpha_{4}=(0.5385,0,0.1569)$.

By Definition 4.1 and 4.2, we have

$s\left(\alpha_{1}\right)=0.4676, s\left(\alpha_{2}\right)=0.6789, s\left(\alpha_{3}\right)=0.5815, s\left(\alpha_{4}\right)=0.6908 ;$

$\alpha_{1}<\alpha_{3}<\alpha_{2}<\alpha_{4}$.

Thus, the alternative $A_{4}$ is the best choice.

Hence, we get exactly the same results as in [31].

Example 4.3 Let us consider the multi-attribute group decision making problem (about the air quality evaluation) adapted from [12]. To evaluate the air quality of Guangzhou for the 16th Asian Olympic Games, the air quality in Guangzhou for the Novembers of 2006, 2007, 2008 and 2009 were collected in order to find out the trends and to forecast the situation in 2010. There are three air-quality monitoring stations which can be seen as decision makers. There are three measured indexes, namely, $\mathrm{SO}_{2}\left(C_{1}\right), \mathrm{NO}_{2}\left(C_{2}\right)$ and $\mathrm{PM}_{10}$ $\left(C_{3}\right)$. The measured values from air-quality monitoring stations under these indexes are shown in Tables 1, 2 and 3 in [12], and they can be expressed by neutrosophic numbers. Let $\left\{S_{1}, S_{2}, S_{3}, S_{4}\right\}=\{$ November of 2006, November of 2007, November of 2008, November of 2009\} be the set of alternatives, please give the rank of air quality from 2006 to 2009 .

According the method in [12], derive the collective overall values:

$r_{1}=(0.288,0.396,0.304)$,

$r_{2}=(0.329,0.344,0.310)$,

$r_{3}=(0.345,0.430,0.210)$,

$r_{4}=(0.417,0.367,0.183)$.

By Definition 4.1 and 4.2, we have

$s\left(r_{1}\right)=0.492, s\left(r_{2}\right)=0.5095, s\left(r_{3}\right)=0.5675, s\left(r_{4}\right)=0.617$;

$r_{1}<r_{2}<r_{3}<r_{4}$.

So, the best alternative is $S_{4}$, i.e., the best air quality Guangzhou is November of 2009 among the Novembers of 2006, 2007, 2008, and 2009. This is in accordance with the conclusion in [12].

\section{The related lattice structure}

In this section, we discuss the lattice structures $\left(D^{*}, \leq_{3}\right)$ and $\left(N S(X), \subseteq_{3}\right)$, where $\leq_{3}$ is defined as follows: $\forall x, y \in D^{*}$,

$$
\begin{aligned}
& x_{3} y \Leftrightarrow\left(\left(x_{1}<y_{1}\right) \text { and }\left(x_{3} \geq y_{3}\right)\right), \\
& \quad \text { or }\left(\left(x_{1}=y_{1}\right) \text { and }\left(x_{3}>y_{3}\right)\right), \\
& \quad \text { or }\left(\left(x_{1}=y_{1}\right) \text { and }\left(x_{3}=y_{3}\right) \text { and }\left(x_{2} \leq y_{2}\right)\right) .
\end{aligned}
$$

Proposition 5.1 Type-3 ordering relation $\leq_{3}$ on $D^{*}$ is a partial ordering relation.

Proof Assume that $x, y, z \in D^{*}$.

i. Reflexivity: $x \leq_{3} x$, it is obvious.

ii. Ant-symmetry: if $x \leq_{3} y$ and $y \leq_{3} x$, then

1. Case 1: $x_{1}<y_{1}$ and $x_{3} \geq y_{3}$. By the definition of $y \leq_{3} x$, we can get $x_{1} \geq y_{1}$, this is contradictory.

2. Case 2: $x_{1}=y_{1}$ and $x_{3}>y_{3}$. By the definition of $y \leq{ }_{3} x$, we can get $x_{3} \leq y_{3}$, it is contradictory.

3. Case 3: $x_{1}=y_{1}$ and $x_{3}=y_{3}$. By $x \leq_{3} y$, we have $x_{2} \leq y_{2}$; also using $y \leq_{3} x$, we have $x_{2} \geq y_{2}$. Thus $x_{2}=y_{2}$.

Summing up the above, if $x \leq_{3} y$ and $y \leq_{3} x$, then $x_{1}=y_{1}$ and $x_{2}=y_{2}$ and $x_{3}=y_{3}$, that is $x=y$.

iii. Transitivity: if $x \leq_{3} y, y \leq_{3} z$, then

1. Case 1: $\left(x_{1}<y_{1}, x_{3} \geq y_{3}\right)$ and $\left(y_{1}<z_{1}, y_{3} \geq z_{3}\right)$. It follows that $x_{1}<z_{1}$ and $x_{3} \geq z_{3}$, thus $x \leq_{3} z$.

2. Case 2: $\left(x_{1}=y_{1}, x_{3}>y_{3}\right)$ and $\left(y_{1}=z_{1}, y_{3}>z_{3}\right)$. It follows that $x_{1}=z_{1}$ and $x_{3}>z_{3}$, thus $x \leq_{3} z$.

3. Case 3: $\left(x_{1}=y_{1}, x_{3}=y_{3}, x_{2} \leq y_{2}\right)$ and $\left(y_{1}=z_{1}, y_{3}=z_{3}\right.$, $\left.y_{2} \leq z_{2}\right)$. It follows that $x_{1}=z_{1}, x_{3}=z_{3}$ and $x_{2} \leq z_{2}$, thus $x \leq{ }_{3} z$.

4. Case 4: $\left(x_{1}<y_{1}, x_{3} \geq y_{3}\right)$ and $\left(y_{1}=z_{1}, y_{3}>z_{3}\right)$. It follows that $x_{1}<z_{1}$ and $x_{3}>z_{3}$, thus $x \leq_{3} z$.

5. Case 5: $\left(x_{1}<y_{1}, x_{3} \geq y_{3}\right)$ and $\left(y_{1}=z_{1}, y_{3}=z_{3}, y_{2} \leq z_{2}\right)$. It follows that $x_{1}<z_{1}, x_{3} \geq z_{3}$ and $y_{2} \leq z_{2}$, thus $x \leq_{3} z$.

6. Case 6: $\left(x_{1}=y_{1}, x_{3}>y_{3}\right)$ and $\left(y_{1}<z_{1}, y_{3} \geq z_{3}\right)$. It follows that $x_{1}<z_{1}$ and $x_{3}>z_{3}$, thus $x \leq_{3} z$.

7. Case 7: $\left(x_{1}=y_{1}, x_{3}>y_{3}\right)$ and $\left(y_{1}=z_{1}, y_{3}=z_{3}, y_{2} \leq z_{2}\right)$. It follows that $x_{1}=z_{1}, x_{3}>z_{3}$ and $y_{2} \leq z_{2}$, thus $x \leq_{3} z$.

8. Case 8: $\left(x_{1}=y_{1}, x_{3}=y_{3}, x_{2} \leq y_{2}\right)$ and $\left(y_{1}<z_{1}, y_{3} \geq z_{3}\right)$. It follows that $x_{1}<z_{1}, x_{3} \geq z_{3}$ and $x_{2} \leq y_{2}$, thus $x \leq_{3} z$.

9. Case 9: $\left(x_{1}=y_{1}, x_{3}=y_{3}, x_{2} \leq y_{2}\right)$ and $\left(y_{1}=z_{1}, y_{3}>z_{3}\right)$, then $x_{1}=z_{1}, x_{3}>z_{3}$ and $x_{2} \leq y_{2}$, thus $x \leq_{3} z$.

Summing up the above, if $x \leq_{3} y$ and $y \leq_{3} z$, then $x \leq_{3} z$. The proof is completed. 
Proposition 5.2 For any $x, y \in D^{*}$, we define

$x \wedge_{3} y=\left\{\begin{array}{l}x, \text { if } x \leq_{3} y \\ y, \text { if } y \leq_{3} x \\ \left(\min \left(x_{1}, y_{1}\right), 1, \max \left(x_{3}, y_{3}\right)\right), \text { otherwise }\end{array}\right.$

$x \vee_{3} y=\left\{\begin{array}{l}y, \text { if } x \leq_{3} y \\ x, \text { if } y \leq_{3} x \\ \left(\max \left(x_{1}, y_{1}\right), 0, \min \left(x_{3}, y_{3}\right)\right), \text { otherwise }\end{array}\right.$

Then $x \wedge_{3} y$ is the greatest lower bound (infimum) of $x$, $y$, denote by $\inf (x, y) ; x \vee_{3} y$ is the least upper bound (supremum) of $x, y$, denote by $\sup (x, y)$. That is, $\left(D^{*}, \leq_{3}\right)$ is a lattice.

Proof If $x \leq_{3} y$ or $y \leq_{3} x$, by the definition of " $\wedge_{3}$ ", $x \wedge_{3} y$ is the greatest lower bound of $x, y$, that is, $x \wedge_{3} y=\inf (x, y)$. Moreover, if $x \leq_{3} y$ or $y \leq_{3} x$, then $x \vee_{3} y$ is the least upper bound of $x, y$, that is, $x \vee_{3} y=\sup (x, y)$.

Now, we suppose that $x \|_{\leq_{3}} y$. Thus, by the definition of " $\wedge_{3}$ " and " $\vee 3$ ",

$x \wedge_{3} y=\left(\min \left(x_{1}, y_{1}\right), 1, \max \left(x_{3}, y_{3}\right)\right)$,

$x \vee_{3} y=\left(\max \left(x_{1}, y_{1}\right), 0, \min \left(x_{3}, y_{3}\right)\right)$.

i. To prove $x \wedge_{3} y=\inf (x, y)$ : Denote

$z=\left(z_{1}, z_{2}, z_{3}\right)=\left(\min \left(x_{1}, y_{1}\right), 1, \max \left(x_{3}, y_{3}\right)\right)$.

Then $x_{1} \geq \min \left(x_{1}, y_{1}\right)=z_{1}, x_{3} \leq \max \left(x_{3}, y_{3}\right)=z_{3}$.

If $x_{1}>z_{1}$ and $x_{3} \leq z_{3}$, then $z \leq_{3} x$.

If $x_{1}=z_{1}$ and $x_{3}<z_{3}$, then $z \leq_{3} x$.

If $x_{1}=z_{1}$ and $x_{3}=z_{3}$, then $y_{1} \geq x_{1}, y_{3} \leq x_{3}$, it follows that $x \leq_{3} y$ or $y \leq_{3} x$. It is a contradiction with the condition $x \|_{\leq_{3}} y$. Hence we can get $z \leq_{3} x$. Similarly, we can get $z \leq_{3} y$. This means that $z$ is the lower bound of $x$ and $y$.

Next, we need prove that $z$ is the greatest lower bound of $x$ and $y$.

Assume $a=\left(a_{1}, a_{2}, a_{3}\right) \in D^{*}$ such that $a \leq_{3} x$ and $a \leq_{3} y$.

1. Case 1: $\left(a_{1}<x_{1}, a_{3} \geq x_{3}\right)$ and $\left(a_{1}<y_{1}, a_{3} \geq y_{3}\right)$. It follows that $a_{1}<\min \left(x_{1}, y_{1}\right)=z_{1}$ and $a_{3} \geq \max \left(x_{3}, y_{3}\right)=z_{3}$, thus $a \leq{ }_{3} z$.

2. Case 2: $\left(a_{1}=x_{1}, a_{3}>x_{3}\right)$ and $\left(a_{1}=y_{1}, a_{3}>y_{3}\right)$. It follows that $a_{1}=\min \left(x_{1}, y_{1}\right)=z_{1}$ and $a_{3}>\max \left(x_{3}, y_{3}\right)=z_{3}$, thus $a \leq{ }_{3} z$.

3. Case 3: $\left(a_{1}=x_{1}, a_{3}=x_{3}, a_{2} \leq x_{2}\right)$ and $\left(a_{1}=y_{1}, a_{3}=y_{3}\right.$, $\left.a_{2} \leq y_{2}\right)$. It follows that $a_{1}=\min \left(x_{1}, y_{1}\right)=z_{1}, a_{3}=\max \left(x_{3}\right.$, $\left.y_{3}\right)=z_{3}$ and $a_{2} \leq \max \left(x_{2}, y_{2}\right) \leq 1=z_{2}$. Thus $a \leq_{3} z$.

4. Case 4: $\left(a_{1}=x_{1}, a_{3}>x_{3}\right)$ and $\left(a_{1}<y_{1}, a_{3} \geq y_{3}\right)$. It follows that $a_{1}=x_{1}=\min \left(x_{1}, y_{1}\right)=z_{1}$ and $a_{3} \geq \max \left(x_{3}, y_{3}\right)=z_{3}$. If $a_{1}=x_{1}=\min \left(x_{1}, y_{1}\right)=z_{1}$ and $a_{3}>\max \left(x_{3}, y_{3}\right)=z_{3}$, then $a \leq_{3} z$; If $a_{1}=x_{1}=\min \left(x_{1}, y_{1}\right)=z_{1}$ and $a_{3}=\max \left(x_{3}\right.$, $\left.y_{3}\right)=z_{3}$, from this and the hidden condition $a_{2} \leq 1=z_{2}$, we get $a \leq_{3} z$.

5. Case 5: $\left(a_{1}<x_{1}, a_{3} \geq x_{3}\right)$ and $\left(a_{1}=y_{1}, a_{3}>y_{3}\right)$. It follows that $a_{1}=y_{1}=\min \left(x_{1}, y_{1}\right)=z_{1}$ and $a_{3} \geq \max \left(x_{3}, y_{3}\right)=z_{3}$. Similar to Case 4, we can get $a \leq_{3} z$.

6. Case 6: $\left(a_{1}<x_{1}, a_{3} \geq x_{3}\right)$ and $\left(a_{1}=y_{1}, a_{3}=y_{3}, a_{2} \leq y_{2}\right)$. It follows that $y_{1}<x_{1}$ and $y_{3} \geq x_{3}$, so $y \leq{ }_{3} x$, it is a contradiction with hypothesis $x \|_{\leq_{3}} y$.

7. Case 7: $\left(a_{1}=x_{1}, a_{3}>x_{3}\right)$ and $\left(a_{1}=y_{1}, a_{3}=y_{3}, a_{2} \leq y_{2}\right)$. It follows that $y_{1}=x_{1}$ and $y_{3}>x_{3}$, so $y \leq{ }_{3} x$, it is a contradiction with hypothesis $x \|_{\leq_{3}}$.

8. Case 8: $\left(a_{1}=x_{1}, a_{3}=x_{3}, a_{2} \leq x_{2}\right)$ and $\left(a_{1}<y_{1}, a_{3} \geq y_{3}\right)$. It follows that $x_{1}<y_{1}$ and $x_{3} \geq y_{3}$, so $x \leq{ }_{3} y$, it is a contradiction with hypothesis $x \|_{\leq_{3}} y$.

9. Case 9: $\left(a_{1}=x_{1}, a_{3}=x_{3}, a_{2} \leq x_{2}\right)$ and $\left(a_{1}=y_{1}, a_{3}>y_{3}\right)$. It follows that $x_{1}=y_{1}$ and $x_{3}>y_{3}$, so $x \leq_{3} y$, it is a contradiction with hypothesis $x \|_{\leq_{3}} y$.

Therefore, we always have $a \leq_{3} z$. That is, $z=\left(\min \left(x_{1}, y_{1}\right)\right.$, $\left.1, \max \left(x_{3}, y_{3}\right)\right)$ is the greatest lower bound of $x, y$.

ii. To prove $x \vee_{3} y=\sup (x, y)$ : Denote

$w=\left(w_{1}, w_{2}, w_{3}\right)=\left(\max \left(x_{1}, y_{1}\right), 0, \min \left(x_{3}, y_{3}\right)\right)$.

Then $x_{1} \leq \max \left(x_{1}, y_{1}\right)=w_{1}, x_{3} \geq \min \left(x_{3}, y_{3}\right)=w_{3}$.

If $x_{1}<w_{1}$ and $x_{3} \geq w_{3}$, then $x \leq_{3} w$.

If $x_{1}=w_{1}$ and $x_{3}>w_{3}$, then $x \leq_{3} w$.

If $x_{1}=w_{1}$ and $x_{3}=w_{3}$, then $y_{1} \leq x_{1}, y_{3} \geq x_{3}$, so $y \leq{ }_{3} x$ or $x \leq_{3} y$, it is a contradiction with hypothesis $x \|_{\leq_{3}} y$. Hence we always have $x \leq{ }_{3} w$.

Similarly, we can prove $y \leq_{3} w$. Therefore, $w$ is an upper bound of $x$ and $y$.

Next, we need prove $w$ is the least upper bound of $x$ and $y$.

Suppose $b=\left(b_{1}, b_{2}, b_{3}\right) \in D^{*}$ such that $x \leq_{3} b, y \leq_{3} b$.

1. Case 1: $\left(x_{1}<b_{1}, x_{3} \geq b_{3}\right)$ and $\left(y_{1}<b_{1}, y_{3} \geq b_{3}\right)$. It follows that $b_{1}>\max \left(x_{1}, y_{1}\right)=w_{1}$ and $b_{3} \leq \min \left(x_{3}, y_{3}\right)=w_{3}$. Thus $w \leq{ }_{3} b$.

2. Case 2: $\left(b_{1}=x_{1}, x_{3}>b_{3}\right)$ and $\left(b_{1}=y_{1}, y_{3}>b_{3}\right)$. It follows that $b_{1}=\max \left(x_{1}, y_{1}\right)=w_{1}, b_{3}<\min \left(x_{3}, y_{3}\right)=w_{3}$, thus $w \leq{ }_{3} b$.

3. Case 3: $\left(b_{1}=x_{1}, b_{3}=x_{3}, x_{2} \leq b_{2}\right)$ and $\left(b_{1}=y_{1}, b_{3}=y_{3}\right.$, $\left.y_{2} \leq b_{2}\right)$. It follows that $b_{1}=\max \left(x_{1}, y_{1}\right)=w_{1}, b_{3}=\min \left(x_{3}\right.$, $\left.y_{3}\right)=w_{3}$ and $b_{2} \geq \max \left(x_{2}, y_{2}\right) \geq 0=w_{2}$, thus $w \leq_{3} b$.

4. Case 4: $\left(b_{1}=x_{1}, x_{3}>b_{3}\right)$ and $\left(y_{1}<b_{1}, y_{3} \geq b_{3}\right)$. It follows that $b_{1} \geq \max \left(x_{1}, y_{1}\right)=w_{1}$ and $b_{3} \leq \min \left(x_{3}, y_{3}\right)=w_{3}$. If $\left(b_{1}>\max \left(x_{1}, y_{1}\right)=w_{1}, b_{3} \leq \min \left(x_{3}, y_{3}\right)=w_{3}\right)$ or $\left(b_{1}=\max \left(x_{1}, y_{1}\right)=w_{1}, b_{3}<\min \left(x_{3}, y_{3}\right)=w_{3}\right)$, then $w \leq_{3} b$; If $b_{1}=\max \left(x_{1}, y_{1}\right)=w_{1}$ and $b_{3}=\min \left(x_{3}, y_{3}\right)=w_{3}$, according the hidden condition $b_{2} \geq 0=w_{2}$, we can get $w \leq_{3} b$.

5. Case 5: $\left(x_{1}<b_{1}, x_{3} \geq b_{3}\right)$ and $\left(b_{1}=y_{1}, y_{3}>b_{3}\right)$. It follows that $b_{1} \geq \max \left(x_{1}, y_{1}\right)=w_{1}$ and $b_{3} \leq \min \left(x_{3}, y_{3}\right)=w_{3}$, similar to Case 4 , we can get $w \leq_{3} b$. 
6. Case 6: $\left(x_{1}<b_{1}, x_{3} \geq b_{3}\right)$ and $\left(b_{1}=y_{1}, b_{3}=y_{3}, y_{2} \leq b_{2}\right)$. It follows that $x_{1}<y_{1}$ and $x_{3} \geq y_{3}$, so $x \leq_{3} y$, it is a contradiction with hypothesis $x \|_{\leq_{3}}$.

7. Case 7: $\left(b_{1}=x_{1}, x_{3}>b_{3}\right)$ and $\left(b_{1}=y_{1}, b_{3}=y_{3}, y_{2} \leq b_{2}\right)$. It follows that $y_{1}=x_{1}$ and $y_{3}<x_{3}$, so $x \leq_{3} y$, it is a contradiction with hypothesis $x \|_{\leq_{3}} y$.

8. Case 8: $\left(b_{1}=x_{1}, b_{3}=x_{3}, x_{2} \leq b_{2}\right)$ and $\left(y_{1}<b_{1}, y_{3} \geq b_{3}\right)$. It follows that $y_{1}<x_{1}$ and $y_{3} \geq x_{3}$, so $y \leq{ }_{3} x$, it is a contradiction with hypothesis $x \|_{\leq_{3}} y$.

9. Case 9: $\left(b_{1}=x_{1}, b_{3}=x_{3}, x_{2} \leq b_{2}\right)$ and $\left(b_{1}=y_{1}, y_{3}>b_{3}\right)$. It follows that $y_{1}=x_{1}$ and $y_{3}>x_{3}$, so $y \leq{ }_{3} x$, it is a contradiction with hypothesis $x \|_{\leq_{3}} y$.

Therefore, we always have $w \leq_{3} b$. This means that $w=\left(\max \left(x_{1}, y_{1}\right), 0, \min \left(x_{3}, y_{3}\right)\right)$ is the least upper bound of $x, y$.

Combing (i) and (ii), we get that $x \wedge_{3} y=\inf (x, y)$, $x \vee_{3} y=\sup (x, y)$, and $\left(D^{*}, \leq_{3}\right)$ is a lattice. The proof is completed.

Definition 5.1 For any $x, y \in D^{*}$, the type- 3 union $x \vee_{3}$ yand type-3 intersection $x \wedge_{3} y$ are defined as Proposition 5.2; the complement is defined as following:

$x^{c}=\left(x_{1}, x_{2}, x_{3}\right)^{c}=\left(x_{3}, 1-x_{2}, x_{1}\right)$.

It is easy to verify the following conclusions.

Proposition 5.3 For any $x, y, z \in D^{*}$, we have

1. $x \wedge_{3} x=x, x \vee_{3} x=x$

2. $x \wedge_{3} y=y \wedge_{3} x, x \vee_{3} y=y \vee_{3} x$

3. $\left(x \wedge_{3} y\right) \wedge_{3} z=x \wedge_{3}\left(y \wedge_{3} z\right),\left(x \vee_{3} y\right) \vee_{3} z=x \vee_{3}\left(y \vee_{3} z\right)$; $x \wedge_{3}\left(y \vee_{3} x\right)=x, x \vee_{3}\left(y \wedge_{3} x\right)=x$

4. $x \leq_{3} y \Leftrightarrow x \vee_{3} y=y ; x \leq_{3} y \Leftrightarrow x \wedge_{3} y=x$;

5. $\left(x^{c}\right)^{c}=x$.

Proposition 5.4 Let $x, y \in D^{*}$, then

1. $\left(x \wedge_{3} y\right)^{c}=x^{c} \vee_{3} y^{c}$

2. $\left(x \vee_{3} y\right)^{c}=x^{c} \wedge_{3} y^{c}$.

Proof Assume that $x=\left(x_{1}, x_{2}, x_{3}\right), y=\left(y_{1}, y_{2}, y_{3}\right) \in D^{*}$. By Definition 5.1, we have

$x^{c}=\left(x_{3}, 1-x_{2}, x_{1}\right), y^{c}=\left(y_{3}, 1-y_{2}, y_{1}\right)$.

If $y \leq{ }_{3} x$, then

Case 1: $y_{1}<x_{1}$ and $y_{3} \geq x_{3}$. It follows that $x^{c} \leq_{3} y^{c}$. Thus $\left(x \vee_{3} y\right)^{c}=y^{c}=x^{c} \vee_{3} y^{c}$.

Case 2: $y_{1}=x_{1}$ and $y_{3}>x_{3}$. It follows that $x^{c} \leq_{3} y^{c}$. $\operatorname{Thus}\left(x \vee_{3} y\right)^{c}=y^{c}=x^{c} \vee_{3} y^{c}$.

Case 3: $y_{1}=x_{1}, y_{3}=x_{3}$ and $y_{2} \leq x_{2}$. It follows that $1-x_{2} \leq 1-y_{2}$. Thus $x^{c} \leq_{3} y^{c}$, and $\left(x \vee_{3} y\right)^{c}=y^{c}=x^{c} \vee_{3} y^{c}$.
Similarly, if $x \leq_{3} y$, then $\left(x \vee_{3} y\right)^{c}=x^{c}=x^{c} \vee_{3} y^{c}$.

If neither $y \leq_{3} x$ nor $x \leq_{3} y$, then

$x \wedge_{3} y=\left(\min \left(x_{1}, y_{1}\right), 1, \max \left(x_{3}, y_{3}\right)\right)$

Thus

$$
\begin{aligned}
\left(x \wedge_{3} y\right)^{c} & =\left(\max \left(x_{3}, y_{3}\right), 0, \min \left(x_{1}, y_{1}\right)\right), \\
x^{c} \vee_{3} y^{c} & =\left(x_{3}, 1-x_{2}, x_{1}\right) \vee_{3}\left(y_{3}, 1-y_{2}, y_{1}\right) \\
& =\left(\max \left(x_{3}, y_{3}\right), 0, \min \left(x_{1}, y_{1}\right)\right) .
\end{aligned}
$$

Hence $\left(x \wedge_{3} y\right)^{c}=x^{c} \vee_{3} y^{c}$.

By (1) and Proposition 5.3 (5), we can get that $\left(x \vee_{3} y\right)^{c}=x^{c} \wedge_{3} y^{c}$.

Theorem 5.1 Denote $1_{D^{*}}=(1,1,0)$ and $0_{D^{*}}=(0,0,1)$. Then $\left(D^{*}, \vee_{3}, \wedge_{3},{ }^{c}, 0_{D *}, 1_{D *}\right)$ is a generalized De Morgan algebra.

Proof By Proposition 5.1-5.4 and Definition 2.10, we can get that $\left(D^{*}, \vee_{3}, \wedge_{3},{ }^{c}, 0_{D *}, 1_{D *}\right)$ is a generalized De Morgan algebra.

The following example shows that $\left(D^{*}, \vee_{3}, \wedge_{3},{ }^{c}, 0_{D *}, 1_{D *}\right)$ is not a De Morgan algebra, since distributive law is not true.

\section{Example 5.1 Let}

$x=(0.2,0.8,0.3)$,

$y=(0.6,0.3,0.4)$,

$z=(0.5,0.9,0.38)$.

Then

$\left(x \wedge_{3} y\right) \vee_{3} z=(0.2,1,0.4) \vee_{3}(0.5,0.9,0.38)=(0.5,0.9,0.38) ;$

$\left(x \vee_{3} z\right) \wedge_{3}\left(y \vee_{3} z\right)=(0.5,0,0.3) \wedge_{3}(0.6,0,0.38)=(0.5,1,0.38)$;

$\left(x \vee_{3} y\right) \wedge_{3} z=(0.6,0,0.3) \wedge_{3}(0.5,0.9,0.38)=(0.5,0.9,0.38)$

$\left(x \wedge_{3} z\right) \vee_{3}\left(y \wedge_{3} z\right)=(0.2,1,0.3) \vee_{3}(0.5,1,0.4)=(0.5,0,0.3)$

Therefore,

$\left(x \wedge_{3} y\right) \vee_{3} z \neq\left(x \vee_{3} z\right) \wedge_{3}\left(y \vee_{3} z\right)$

$\left(x \vee_{3} y\right) \wedge_{3} z \neq\left(x \wedge_{3} z\right) \vee_{3}\left(y \wedge_{3} z\right)$

This means that the distributive law is not satisfied in the generalized De Morgan algebra $\left(D^{*}, \vee_{3}, \wedge_{3},{ }^{c}, 0_{D *}, 1_{D *}\right)$.

By Definition 4.1 and Proposition 3.2, we have

For any neutrosophic sets $A$ and $B$ in $X$, by Definition 3.1 we have

$A \subseteq_{3} B$ if and only if

$\left(T_{A}(x), I_{A}(x), F_{A}(x)\right) \leq_{3}\left(T_{B}(x), I_{B}(x), F_{B}(x)\right), \forall x \in X$. 
From this, using Proposition 5.1, we can easy to prove Proposition 3.1.

Moreover, for any neutrosophic sets $A$ and $B$ in $X$, by Definition 3.2 and 5.1 we have

$A \cup_{3} B=\left\{\left(T_{A}(x), I_{A}(x), F_{A}(x)\right) \vee_{3}\left(T_{B}(x), I_{B}(x), F_{B}(x)\right) \mid x \in X\right\} ;$ $A \cap_{3} B=\left\{\left(T_{A}(x), I_{A}(x), F_{A}(x)\right) \wedge_{3}\left(T_{B}(x), I_{B}(x), F_{B}(x)\right) \mid x \in X\right\}$.

From this, using Proposition 5.2-5.4, we can easy to prove Proposition 3.2-3.4. It follows that Theorem 3.1 is a corollary of Theorem 5.1.

Finally, from Example 5.1 we can get that $\left(N S(X), \cup_{3}, \cap_{3}\right)$ is not a distribute lattice, that is, there exits $A, B, C$ in $N S(X)$ such that

$\left(A \cap_{3} B\right) \cup_{3} C \neq\left(A \cup_{3} C\right) \cap_{3}\left(B \cup_{3} C\right)$,

$\left(A \cup_{3} B\right) \cap_{3} C \neq\left(A \cap_{3} C\right) \cup_{3}\left(B \cap_{3} C\right)$.

This result shows that neutrosophic set is different from the fuzzy set and intuitionistic fuzzy set from the algebraic structure.

\section{Conclusion}

In this paper, the inclusion relation between neutrosophic sets is deeply analyzed and studied. Two basic definitions of inclusion relation (type- 1 and type-2 inclusion relations) are summarized, and their shortcomings are pointed out. The definition of type- 3 inclusion relation of neutrosophic sets is proposed. The difference between type- 3 and type- 1 (type-2) inclusion relation is mainly reflected in the processing method of second component of neutrosophic set. For the original definitions (type- 1 and type-2), the second component of neutrosophic set is rigidly assigned to first or third components, which does not really reflect the original meaning of second component. The type- 3 inclusion relation proposed in this paper overcomes this shortcoming.

Moreover, applying the new inclusion relation and its basic idea, this paper also proposes a new ranking method of neutrosophic sets, and illustrates its application in multiattribute decision making by some examples.

Finally, based on type-3 inclusion relation, the lattice structure of neutrosophic set is analyzed, the type- 3 union and intersection operations are established, and the basic algebraic properties are proved. In particular, we get the following important conclusions: all of neutrosophic sets in a certainty universe along with type- 3 union, type- 3 intersection and the complement constitute a generalized De Morgan algebra (non-distribute), which in theory clearly distinguishes the essential difference between neutrosophic set and fuzzy set (intuitionistic fuzzy set), since one can obtain distributive De Morgan algebras for fuzzy set, intuitionistic fuzzy set and so on. This can be seen as a meaningful result of this paper.

Acknowledgements This work was supported by National Natural Science Foundation of China (Grant nos. 61573240, 61473239).

\section{References}

1. Bo CX, Zhang XH (2017) New operations of picture fuzzy relations and fuzzy comprehensive evaluation. Symmetry 9(11):268. https://doi.org/10.3390/sym9110268,

2. Borzooei RA, Farahani H, Moniri M (2014) Neutrosophic deductive filters on BL-algebras. J Intell Fuzzy Syst 26:2993-3004

3. Crispin Sweety CA, Arockiarani I (2017) Rough sets in neutrosophic approximation space. Ann Fuzzy Math Inform 13(4):449-463

4. Cuong BC (2014) Picture fuzzy sets. J Comput Sci Cybern 30(4):409-420

5. Cuong BC, Hai PV (2015) Some fuzzy logic operators for picture fuzzy sets. In: IEEE seventh international conference on knowledge and systems engineering, pp 132-137

6. Cuong BC, Phong PH, Smarandache F (2016) Standard neutrosophic soft theory: some first results. Neutrosophic Sets Syst 12:80-91

7. Chen JQ, Ye J (2017) Some single-valued neutrosophic Dombi weighted aggregation operators for multiple attribute decisionmaking. Symmetry 9:82. https://doi.org/10.3390/sym9060082

8. Guo YH, Şengür A, Ye J (2014) A novel image thresholding algorithm based on neutrosophic similarity score. Measurement 58:175-186

9. Jun YB, Smarandache F, Kim CS (2017) Neutrosophic cubic sets. New Math Nat Comput 13(1):41-54

10. Jun YB, Smarandache F, Kim CS (2017) P-union and P-intersection of neutrosophic cubic sets. Analele Universitatii "Ovidius" Constanta Seria Matematica 25(1):99-115

11. Liu PD, Li HG (2017) Multiple attribute decision-making method based on some normal neutrosophic Bonferroni mean operators. Neural Comput Appl 28(1):179-194

12. Liu PD, Chu YC, Li YW, Chen YB (2014) Some generalized neutrosophic number Hamacher aggregation operators and their application to group decision making. Int J Fuzzy Syst 16(2):242-255

13. Liu YL, Yang HL (2017) Further research of single valued neutrosophic rough sets. J Intell Fuzzy Syst 33:1467-1478

14. Nguyen GN, Son LH, Ashour AS, Dey N (2017) A survey of the state-of-the-arts on neutrosophic sets in biomedical diagnoses. Int J Mach Learn Cybern. https://doi.org/10.1007/s13042-017-06917. (Online)

15. Peng JJ, Wang JQ, Zhang HY, Chen XH (2014) An outranking approach for multi-criteria decision-making problems with simplified neutrosophic sets. Appl Soft Comput 25:336-346

16. Peng JJ, Wang JQ, Wang J, Zhang HY, Chen XH (2016) Simplified neutrosophic sets and their applications in multi-criteria group decision-making problems. Int J Syst Sci 47(10):2342-2358

17. Rezaei A, Saeid AB, Smarandache F (2015) Neutrosophic filters in BE-algebras. Ratio Math 29:65-79

18. Singh PK (2017) Three-way fuzzy concept lattice representation using neutrosophic set. Int J Mach Learn Cybern 8(1):69-79

19. Sevcovic D (1996) Free non-distributive Morgan-Stone algebras. N Z J Math 25:85-94

20. Smarandache F (1998) Neutrosophy, neutrosophic probability, set, and logic. American Research Press, Rehoboth

21. Smarandache F (2005) Neutrosophic set-a generialization of the intuitionistics fuzzy sets. Int J Pure Appl Math 24(3):287-297 
22. Smarandache F (2017) Neutrosophic perspectives: triplets, duplets, multisets, hybrid operators, modal logic, hedge algebras and applications. Pons Publishing House, Brussels

23. Wang C, Ha MH, Liu XW (2015) A mathematical model of ternary fuzzy set for voting. J Intell Fuzzy Syst 29(6):2381-2386

24. Wang H, Smarandache F, Zhang YQ et al (2010) Single valued neutrosophic sets. Multispace Multistruct Neutrosophic Transdiscipl 4:410-413

25. Yang HL, Zhang CL, Guo ZL, Liu YL, Liao XW (2017) A hybrid model of single valued neutrosophic sets and rough sets: single valued neutrosophic rough set model. Soft Comput 21(21):6253-6267

26. Yao YY (2010) Three-way decisions with probabilistic rough sets. Inf Sci 180:341-353

27. Yao YY (2017) Interval sets and three-way concept analysis in incomplete contexts. Int J Mach Learn Cybern 8(1):3-20

28. Ye J (2015) Improved cosine similarity measures of simplified neutrosophic sets for medical diagnoses. Artif Intell Med 63:171-179

29. Ye J (2016) Projection and bidirectional projection measures of single-valued neutrosophic sets and their decision-making method for mechanical design schemes. J Exp Theor Artif Intell 29(4):731-740

30. Ye J (2016) Fault diagnoses of steam turbine using the exponential similarity measure of neutrosophic numbers. J Intell Fuzzy Syst 30(4):1927-1934

31. Ye J (2014) A multicriteria decision-making method using aggregation operators for simplified neutrosophic sets. J Intell Fuzzy Syst 26(5):2459-2466
32. Ye J, Du S (2017) Some distances, similarity and entropy measures for interval-valued neutrosophic sets and their relationship. Int J Mach Learn Cybern. https://doi.org/10.1007/s1304 2-017-0719-z

33. Ye J (2017) Simplified neutrosophic harmonic averaging projection-based method for multiple attribute decision making problems. Int J Mach Learn Cybern 8(3):981-987

34. Zhan JM, Khan M, Gulistan M, Ali A (2017) Applications of neutrosophic cubic sets in multi-criteria decision-making. Int J Uncertain Quantif 7(5):377-394

35. Zhang XH (2017) Fuzzy anti-grouped filters and fuzzy normal filters in pseudo-BCI algebras. J Intell Fuzzy Syst 33:1767-1774

36. Zhang XH, Ma YC, Smarandache F (2017) Neutrosophic regular filters and fuzzy regular filters in pseudo-BCI algebras. Neutrosophic Sets Syst 17:10-15

37. Zhang XH, Smarandache F, Liang XL (2017) Neutrosophic duplet semi-group and cancellable neutrosophic triplet groups. Symmetry 9(11):275. https://doi.org/10.3390/sym9110275

38. Zhang XH, Yu P, Smarandache F, Park C (2018) Redefined neutrosophic filters in BE-algebras. Ital J Pure Appl Math (in press)

39. Zhang XH, Smarandache F, Ali M, Liang XL (2018) Commutative neutrosophic triplet group and neutro-homomorphism basic theorem. Ital J Pure Appl Math (in press)

Publisher's Note Springer Nature remains neutral with regard to jurisdictional claims in published maps and institutional affiliations. 